\title{
Multiple Linear Cryptanalysis of a Reduced Round RC6
}

\author{
Takeshi Shimoyama, Masahiko Takenaka, and Takeshi Koshiba \\ Secure Computing Lab., Fujitsu Laboratories Ltd., \\ 4-1-1, Kamikodanaka Nakahara-ku Kawasaki 211-8588, Japan \\ \{shimo, takenaka, koshiba\}@flab.fujitsu.co.jp
}

\begin{abstract}
In this paper, we apply multiple linear cryptanalysis to a reduced round RC6 block cipher. We show that 18-round RC6 with weak key is breakable by using the multiple linear attack.
\end{abstract}

\section{Introduction}

The block cipher RC6 was proposed by Rivest et al. in [17] to meet the requirements of the Advanced Encryption Standard (AES) and is one of the finalists of the AES candidates. It has been admired for its high-level security and highspeed software implementation especially on Intel CPU. RC6 enters also the NESSIE Project selection and it has been nominated to the Phase II evaluation.

RC6 is designed based on the block cipher RC5 [16] which makes essential use of arithmetic key additions and data-dependent rotations. Kaliski and Robshaw [7] evaluated the resistance of RC5, which introduced data-dependent rotations as primitive operations, against Linear Attack [14. Borst, Preneel, and Vandewalle [2] refined the linear attack of RC5. As additional primitive operations to RC6, the inclusion of arithmetic multiplications and fixed rotations is believed to contribute the strength of the security of RC6. There are some cryptanalyses of RC6: resistance against Differential Attack, Linear Attack, and Related Key Attack by Contini et al. 3], Mod $n$ Attack [11, Linear Attack [2], and Statistical Attack [5]. One of most effective attacks is $\chi^{2}$ attack by Knudsen and Meier [13] which can break up to 15-round RC6 with general keys and 17-round RC6 with weak keys. We note that their estimation is inferred from experimental results for at most 6-round RC6 and is not relied on any theoretical evidence. The cryptanalysis by Contini et al. [4] is actually is not only of RC6 itself but also of reduced variants of RC6. We enumerate attacks on RC6 in Table 1

In [3, Contini et al. showed some upper bound of complexity to break RC6 against the linear attack on the assumption that the attacker uses the bias of the linear equations with respect to 1-bit masks both on input and output to arithmetic additive operations and the number of equations among multiple linear approximation, which are derived based on the notion of "linear hulls", to advantage.

In this paper, we evaluate the resistance of RC6 with 256-bit key against multiple linear attack. In order to do this, we use the technique of the linear 
Table 1. Attacks on RC6

\begin{tabular}{lccl}
\hline \multicolumn{1}{c}{ Attack } & Rounds & Data size & Comments \\
\hline $\begin{array}{l}\text { Linear Attack [2] } \\
\text { Differential Attack [3] }\end{array}$ & 16 & $2^{119}$ & Upper bound of complexity \\
$\begin{array}{l}\text { Mod } n \text { Cryptanalysis [1] } \\
\chi^{2} \text { Cryptanalysis [13] }\end{array}$ & 12 & $2^{117}$ & Upper bound of complexity \\
& 15 & $2^{119.0}$ & $\begin{array}{l}\text { Lower bound of complexity } \\
\text { (estimation) }\end{array}$ \\
$\begin{array}{l}\text { Multiple Linear Attack } \\
\text { [This paper] }\end{array}$ & 18 & $\leq 2^{118}$ & $\begin{array}{l}\text { Lower bound } \\
\text { (estimation, } 1 / 2^{80} \text { weak keys) }\end{array}$ \\
\hline
\end{tabular}

probability that we obtain by taking multiple paths into account and the theory of multiple linear approximation and evaluate rigorously the complexity to break RC6. To do that, we introduce a novel technique to use a "Matrix Representation" that is a generalization of the piling up lemma to obtain the linear probability. This technique ease us to count the multiple path and to estimate more exactly the linear probability that might depend on the extended-keys. As a result, we show that the target key of 14-round RC6 can be recovered and also that the target key of 18-round RC6 with weak keys, which exists with probability $1 / 2^{90}$ at least, can be recovered.

\section{Preliminary}

For any function $Y=F(X)$, input mask $\Gamma X$ and output mask $\Gamma Y$, we define the bias of linear equations $\operatorname{Bias}_{F}()$ and the linear probability $L P_{F}()$ as follows.

$$
\begin{aligned}
& \operatorname{Bias}_{F}(\Gamma X \rightarrow \Gamma Y)=2 \cdot \frac{\#\{X \mid(\Gamma X \cdot X) \oplus(\Gamma Y \cdot F(X))=0\}}{\#\{X\}}-1 \\
& L P_{F}(\Gamma X \rightarrow \Gamma Y)=\left(\operatorname{Bias}_{F}(\Gamma X \rightarrow \Gamma Y)\right)^{2}
\end{aligned}
$$

It is well known that for any $r$ functions $X_{i+1}=F_{i}\left(X_{i}\right)(i=1, \ldots, r)$ the composite function $H(X)=F_{r} \circ \cdots \circ F_{1}(X)$ has the expected (w.r.t. keys) linear probability satisfying

$$
L P_{H}\left(\Gamma X_{1} \rightarrow \Gamma X_{r+1}\right)=\sum_{\Gamma X_{2}, \ldots, \Gamma X_{r}}\left\{\prod_{i=1}^{r} L P_{F_{i}}\left(\Gamma X_{i} \rightarrow \Gamma X_{i+1}\right)\right\}
$$

Let $e_{0}, \ldots, e_{31}$ denote unit vectors over $G F(2)^{32}$ such that the $i$ th element of $e_{i}$ is 1 and the other elements of $e_{i}$ are 0 . Here we adopt the description of the descending order for vectors (e.g., $\left.e_{0}=(0, \ldots, 0,1)\right)$.

In this paper, we identify 32-bit values, which are used in RC6 encryption, with elements of $G F(2)^{32}$, unless otherwise specified.

\footnotetext{
${ }^{1}$ Strictly speaking, we need more structural information of functions $F_{i}$ for holding the equation.
} 

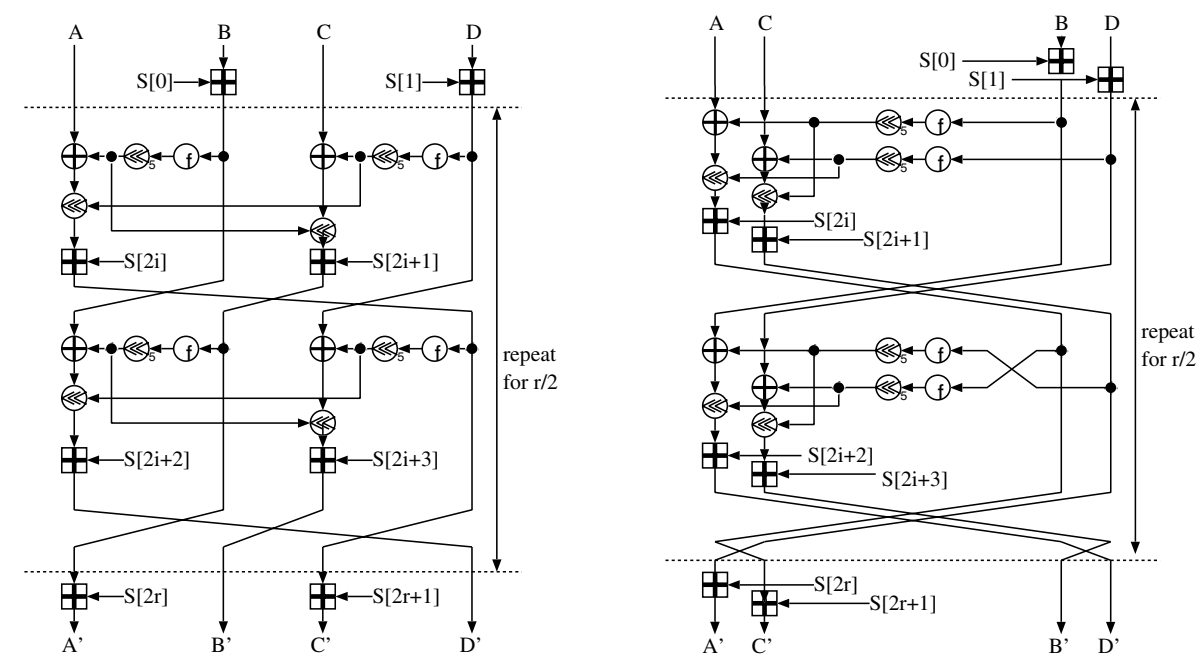

Fig. 1. Original RC6 and its equivalent transformation

\section{$3 \quad$ RC6 and Its Equivalent Transformation}

RC6 is a block cipher proposed by Rivest et al. [17]. It has a variable number of rounds denoted $r$ and key size of $8 b$ bits. The design is word-oriented for word sizes $w$ and the block size is $4 w$. Currently RC6 with $r=20,4 w=128$ and $8 b=128,192,256$ is recommended to give sufficient resistance against possible attacks. (See Figure 1). In this paper, we refer $n$-round RC6 to RC6 $6_{(n)}$. We leave the key scheduling of RC6, which generates extended keys from private keys, out of consideration.

In this paper, we use a Feistel-like description of RC6 which is obtained by exchanging input-output words $B$ and $C$ equivalently. It is easy to see that the new description help us to capture structural properties of RC6. (As long as the authors know, the new description is not shown.)

We consider a block cipher $\mathrm{RC} 6 \oplus$ that is obtained by replacing arithmetic additions of extended-keys of RC6 by exclusive-oring of extended-keys.

Moreover, we consider weak-keys of $2 r$-round RC6. We define two types of weak keys. "Type I weak keys" are ones such that $l s b_{5}(S[4 i-3])=l s b_{5}(S[4 i-$ $4])=0$, and "Type II weak keys" are ones such that $l s b_{4}(S[4 i-3])=l s b_{4}(S[4 i-$ $4])=0$. It is easy to see that Type I weak-keys is of the fraction $2^{-10 r}$, and Type II weak-keys is of the fraction $2^{-8 r}$. Later, we will show that those of weak-keys are actually "weak". 


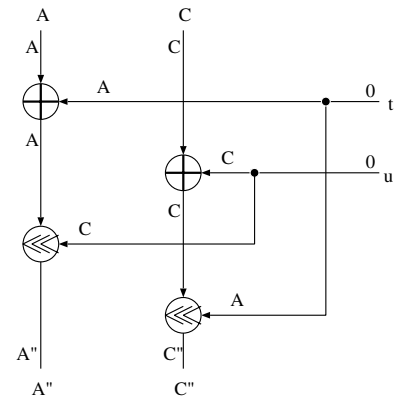

Fig. 2. Linear mask of $R$

\section{Linear Probabilities of Data-Dependent Rotation}

We consider a partial function $R$ of $R C 6$ as follows. (See also Figure 2).

$$
\begin{gathered}
R:(A, C, t, u) \in\left(G F(2)^{32}\right)^{4} \rightarrow\left(A^{\prime \prime}, C^{\prime \prime}\right) \in\left(G F(2)^{32}\right)^{2} . \\
A^{\prime \prime}=(A \oplus t) \ll< \\
C^{\prime \prime}=(C \oplus u) \ll \ll t
\end{gathered}
$$

Let $\Gamma A, \Gamma C, \Gamma t, \Gamma u, \Gamma A^{\prime \prime}, \Gamma A^{\prime \prime}$ be masks for the variables $A, C, t, u, A^{\prime \prime}, C^{\prime \prime}$ of $R$, respectively. We consider linear approximation with significant linear probability such that $\Gamma t=\Gamma u=0$.

Let us consider, for example, the case where $\Gamma A=\Gamma C=\Gamma A^{\prime \prime}=\Gamma C^{\prime \prime}=e_{0}$. Then, if $l s b_{5}(t)=l s b_{5}(u)=0$ then $A e_{0} \oplus A^{\prime \prime} e_{0}=0$ and $C e_{0} \oplus C^{\prime \prime} e_{0}=0$. Only if the case where $l s b_{5}(t)=l s b_{5}(u)=0$ occurs (its probability is $2^{-10}$ ), the probability that the equation $A e_{0} \oplus C e_{0} \oplus A^{\prime \prime} e_{0} \oplus C^{\prime \prime} e_{0}=0$ holds is biased. Thus, we have

$$
L P_{R}\left(\left(e_{0}, e_{0}, 0,0\right) \rightarrow\left(e_{0}, e_{0}\right)\right)=2^{-20} .
$$

Similarly, we obtain the following equations for $i, j, k, l \in\{0,1,2,3,4\}$

$$
L P_{R}\left(\left(e_{i}, e_{j}, 0,0\right) \rightarrow\left(e_{k}, e_{l}\right)\right)=2^{-20}
$$

\section{Linear Probability of RC6 with Weak Keys}

In this section, we consider the linear probability of $2 r$-round RC6 with Type II weak keys by taking "multiple paths". In general, the linear characteristic probability depends on the key-value. Here, for simplicity, we consider RC6 $\oplus$. We assume that key is randomly distributed. In the case that the least significant five bits of extended-key related to linear approximation is fixed (especially, in the case of weak-keys), we can calculate the precise linear probability for each linear approximation. We will discuss how to calculate it in Section 7. 
Table 2. 4-round multiple linear path of $\mathrm{RC} 6 \oplus$

\begin{tabular}{|c||ccc|}
\hline round & output mask of $(A, C)$ \\
\hline 0 & \multicolumn{3}{|c|}{$\left(e_{i}, e_{j}\right)$} \\
& $\swarrow$ & $\ldots$ & $\searrow$ \\
2 & $\left(e_{0}, e_{0}\right)$ & $\ldots$ & $\left(e_{4}, e_{4}\right)$ \\
& $\searrow$ & $\ldots$ & $\swarrow$ \\
4 & & $\left(e_{k}, e_{l}\right)$ & \\
\hline$L P$ & $2^{-40}$ & $\ldots$ & $2^{-40}$ \\
\hline
\end{tabular}

Let us consider 4-round $\mathrm{RC} 6 \oplus$. If we set the input mask for $(A, C)$ being $\left(e_{i}, e_{j}\right)(i, j \in\{0, \ldots, 4\})$ and its output mask being $\left(e_{k}, e_{l}\right)(k, l \in\{0, \ldots, 4\})$, then for any $i, j, s, t \in\{0, \ldots, 4\}$ the following holds:

$$
\left|\operatorname{Bias}_{R}\left(\left(e_{i}, e_{j}, 0,0\right) \rightarrow\left(e_{s}, e_{t}\right)\right)\right|=2^{-10} .
$$

Thus we can show that the absolute value of linear characteristic per path of 4-round $\mathrm{RC} 6 \oplus$ is $2^{-40}$ by the piling up lemma in average of the key.

Since there exist at least $25=5^{2}$ linear characteristic paths such that input mask $\left(e_{i}, e_{j}\right)$ and output mask $\left(e_{k}, e_{l}\right)$ are equal but any other intermediate masks are different from the input mask, we can calculate linear characteristic over multiple paths. (See Table 2.)

$$
\begin{aligned}
& L P_{R C 6 \oplus_{(4)}}\left(\left(e_{0}, e_{0}, 0,0\right) \rightarrow\left(e_{0}, e_{0}, 0,0\right)\right) \\
& =\sum_{s, t} L P_{R}\left(\left(e_{0}, e_{0}\right) \rightarrow\left(e_{s}, e_{t}\right)\right) L P_{R}\left(\left(e_{s}, e_{t}\right) \rightarrow\left(e_{0}, e_{0}\right)\right) \\
& =25 \cdot\left(2^{-40}\right)=2^{-35.356}
\end{aligned}
$$

Moreover, the linear probability of $2 r$-round $\mathrm{RC} 6 \oplus$ is derived as follows.

$$
\begin{aligned}
L P_{R C 6} & \oplus_{(2 r)}\left(\left(e_{i}, e_{j}, 0,0\right) \rightarrow\left(e_{k}, e_{l}, 0,0\right)\right) \\
& \geq 2^{-20}\left(25 \cdot\left(2^{-20}\right)\right)^{r-1} \\
& =2^{-20-15.356(r-1)}
\end{aligned}
$$

It is easy to consider the linear approximation of $(2 r+1)$-round $\mathrm{RC} 6 \oplus$ from the linear approximation of $2 r$-round RC $6 \oplus$ obtained above. (See Table 3 )

Next, we consider Type II weak-keys of RC6. It is easy to see that if $l s b_{4}(K)=$ 0 then arithmetic addition of some fixed 32-bit value $K$ (say, $Y=X+K \bmod$ $2^{32}$ ) does not cause any carry-over in the least significant 5 bits. In this case, the equation $L P_{a d d_{K}}\left(e_{i} \rightarrow e_{i}\right)=1$ always holds for $i \in\{0, \ldots, 4\}$. Such keys can be generated with probability $2^{-4}$ if $K$ is randomly distributed.

This implies that the linear probability of RC6 with weak key of Type II is independent of keys. Thus, we can say that the resistance of RC6 with such keys against multiple linear attack is reduced to the one of $\mathrm{RC} 6 \oplus$ against multiple linear attack. In this sense, we can regard such keys as weak ones. (For example, some weak keys of 3-round RC6 are characterized as ones with least significant four bits each of $S[0], S[1], S[4], S[5]$ is 0 . So, the fraction of such weak keys is $2^{-16}$.) 
Table 3. The linear probability of RC6 with Type II weak keys (or RC6甲)

\begin{tabular}{ccccc}
\hline round & $\begin{array}{c}\text { input } \\
\text { mask }\end{array}$ & $\begin{array}{c}\text { output } \\
\text { mask }\end{array}$ & $\begin{array}{c}\text { linear } \\
\text { probability }\left(\log _{2}\right)\end{array}$ & $\begin{array}{c}\text { fraction of } \\
\text { weak keys of RC6 }\end{array}$ \\
\hline 3 & $\left(0,0, e_{i}, e_{j}\right)$ & $\left(e_{k}, e_{l}, 0,0\right)$ & -20.000 & $2^{-16}$ \\
5 & $\left(0,0, e_{i}, e_{j}\right)$ & $\left(e_{k}, e_{l}, 0,0\right)$ & -35.356 & $2^{-24}$ \\
7 & $\left(0,0, e_{i}, e_{j}\right)$ & $\left(e_{k}, e_{l}, 0,0\right)$ & -50.712 & $2^{-32}$ \\
9 & $\left(0,0, e_{i}, e_{j}\right)$ & $\left(e_{k}, e_{l}, 0,0\right)$ & -66.068 & $2^{-40}$ \\
11 & $\left(0,0, e_{i}, e_{j}\right)$ & $\left(e_{k}, e_{l}, 0,0\right)$ & -81.424 & $2^{-48}$ \\
13 & $\left(0,0, e_{i}, e_{j}\right)$ & $\left(e_{k}, e_{l}, 0,0\right)$ & -96.780 & $2^{-56}$ \\
15 & $\left(0,0, e_{i}, e_{j}\right)$ & $\left(e_{k}, e_{l}, 0,0\right)$ & -112.136 & $2^{-64}$ \\
17 & $\left(0,0, e_{i}, e_{j}\right)$ & $\left(e_{k}, e_{l}, 0,0\right)$ & -127.492 & $2^{-72}$ \\
\hline \multicolumn{5}{c}{ Note that $i, j, k, l$ range over $\{0, \ldots, 4\}}$.
\end{tabular}

Table 4. The linear probability of 2-round RC6

\begin{tabular}{c|cccc}
\hline Input mask $(\Gamma A, \Gamma C)$ & \multicolumn{4}{|c}{$\left(e_{i}, e_{j}\right), i, j \in\{0, \ldots, 4\}$} \\
Output mask $\left(\Gamma A^{\prime}, \Gamma C^{\prime}\right)$ & $\left(e_{0}, e_{0}\right)$ & $\left(e_{k}, e_{0}\right)$ & $\left(e_{0}, e_{l}\right)$ & $\left(e_{k}, e_{l}\right)$ \\
Linear prob. of addition & 1 & $2^{-2}$ & $2^{-2}$ & $2^{-4}$ \\
Linear prob. of 1-round RC6 & $2^{-20}$ & $2^{-22}$ & $2^{-22}$ & $2^{-24}$ \\
\hline Note that $k \neq 0, l \neq 0$.
\end{tabular}

\section{Linear Probability of RC6}

There are several researches about success probability of linear approximation for arithmetic addition $Y=X+K$ on the assumption that $K$ is randomly chosen but fixed ([710 154]). In this paper, we consider linear approximation only of the form $X e_{i} \oplus Y e_{i}=0$, which is a relation between a 1-bit of input and a 1-bit of output. Let us see it more precisely.

It is well known that the expectation (w.r.t. keys) of the bias of linear equations satisfies that $L P_{a d d_{K}}\left(e_{0} \rightarrow e_{0}\right)=1$, and $L P_{a d d_{K}}\left(e_{i} \rightarrow e_{i}\right)=2^{-2},(i \neq 0)$ on the average of $K$. By utilizing these equations, it is easy to calculate the linear probability of 2-round RC6 with the key addition. We note that the linear probability of key addition can be obtained only from output masks. The linear probability of 2-round RC6 follows from the linear probability of addition, the linear probability of 1-round $\mathrm{RC} 6 \oplus$, obtained in the previous section, and the piling up lemma. (See Table 4)

Any output mask $\left(\Gamma A^{\prime}, \Gamma C^{\prime}\right)$ corresponds with one of $\left(e_{0}, e_{0}\right),\left(e_{k}, e_{0}\right),\left(e_{0}, e_{l}\right)$ and $\left(e_{k}, e_{l}\right)$. The number of output masks of each type is $1,4,4$ and 16 , respectively. Thus we have the linear probability of 4-round RC6 over multiple paths such that the input mask is of the form $\left(e_{i}, e_{j}, 0,0\right)$ and the output mask is of the form $\left(e_{0}, e_{0}, 0,0\right)$ as follows.

$$
\begin{aligned}
& L P_{R C 6_{(4)}}\left(\left(e_{i}, e_{j}, 0,0\right) \rightarrow\left(e_{0}, e_{0}, 0,0\right)\right) \\
& =2^{-20}\left(2^{-20}+4 \cdot 2^{-22}+4 \cdot 2^{-22}+16 \cdot 2^{-24}\right) \\
& =2^{-38}
\end{aligned}
$$


Table 5. The linear probability of RC6

\begin{tabular}{cccc}
\hline round & $\begin{array}{c}\text { input mask } \\
(A, C, B, D)\end{array}$ & $\begin{array}{c}\text { output mask } \\
\left(\mathrm{A}^{\prime}, \mathrm{C}^{\prime}, \mathrm{B}^{\prime}, \mathrm{D}^{\prime}\right)\end{array}$ & $\begin{array}{c}\text { linear probability } \\
\left(\log _{2}\right)\end{array}$ \\
\hline 3 & $\left(0,0, e_{i}, e_{j}\right)$ & $\left(e_{k}, e_{l}, 0,0\right)$ & $-20-2 \mu(\{i, j, k, l\})$ \\
5 & $\left(0,0, e_{i}, e_{j}\right)$ & $\left(e_{k}, e_{l}, 0,0\right)$ & $-38-2 \mu(\{i, j, k, l\})$ \\
7 & $\left(0,0, e_{i}, e_{j}\right)$ & $\left(e_{k}, e_{l}, 0,0\right)$ & $-56-2 \mu(\{i, j, k, l\})$ \\
9 & $\left(0,0, e_{i}, e_{j}\right)$ & $\left(e_{k}, e_{l}, 0,0\right)$ & $-74-2 \mu(\{i, j, k, l\})$ \\
11 & $\left(0,0, e_{i}, e_{j}\right)$ & $\left(e_{k}, e_{l}, 0,0\right)$ & $-92-2 \mu(\{i, j, k, l\})$ \\
13 & $\left(0,0, e_{i}, e_{j}\right)$ & $\left(e_{k}, e_{l}, 0,0\right)$ & $-110-2 \mu(\{i, j, k, l\})$ \\
\hline \multicolumn{5}{c}{ Note that $i, j, k, l \in\{0, \ldots, 4\}, \mu(X)=\#\{x \neq 0 \mid x \in X\}}$.
\end{tabular}

Similarly, we have the linear probability of $2 r$-round RC6 over the same multiple paths as follows.

$$
L P_{R C 6_{(2 r)}}\left(\left(e_{i}, e_{j}, 0,0\right) \rightarrow\left(e_{0}, e_{0}, 0,0\right)\right) \geq 2^{-20-18(r-1)}
$$

Furthermore, we have the linear probability of $2 r$-round RC6 over multiple paths of the other types.

$$
\begin{aligned}
& L P_{R C 6_{(2 r)}}\left(\left(e_{i}, e_{j}, 0,0\right) \rightarrow\left(e_{k}, e_{0}, 0,0\right)\right) \geq 2^{-22-18(r-1)} \\
& L P_{R C 6_{(2 r)}}\left(\left(e_{i}, e_{j}, 0,0\right) \rightarrow\left(e_{0}, e_{l}, 0,0\right)\right) \geq 2^{-22-18(r-1)} \\
& L P_{R C 6_{(2 r)}}\left(\left(e_{i}, e_{j}, 0,0\right) \rightarrow\left(e_{k}, e_{l}, 0,0\right)\right) \geq 2^{-24-18(r-1)}
\end{aligned}
$$

By utilizing the linear approximation of $2 r$-round RC6, it is not hard to consider the linear approximation of $2 r+1$-round RC6, We note that the linear probability is affected by the extended-key that is added to input data $B$ and $D$ to the first round. Namely, we have to take into account that the linear probability depends on the bit position of the input mask. We illustrate an estimation of the linear probability of reduced-round RC6 in Table 5

\section{Linear Probability of a Fixed Key}

In this section, we give a way to calculate the more precise linear probability of the linear approximation $A e_{i} \oplus C e_{j} \oplus A^{\prime} e_{k} \oplus C^{\prime} e_{l}=0$ for RC6 with any fixed key. In Section 5 and Section 6 , we calculated the linear probability in average of keys.

On the other hand, especially as in the case of Type I weak keys, that is the least significant five bits of the extended key is 0 , then, by keeping the sign of the bias of linear equation in mind when summing the bias of linear equation, we can generalize the piling up lemma to calculate the bias of linear equation more precisely.

Now, we consider the linear probability of RC6 with Type I weak key. For a simpler exposition, we calculate the linear probability of 4-round RC6 with the weak keys such that the input and the output mask are both $\left(e_{0}, e_{0}, 0,0\right)$. We show the bias of each linear characteristic in Table 6. For example, the linear characteristic for paths from the 0th round through the second round to the fourth round trace $\left(e_{0}, e_{0}\right) \rightarrow\left(e_{1}, e_{1}\right) \rightarrow\left(e_{0}, e_{0}\right)$ is $\left(-2^{-10}\right) \cdot\left(-2^{-10}\right)=+2^{-20}$. 
Table 6. The bias of linear characteristic path such that the input and output masks are both $\left(e_{0}, e_{0}, 0,0\right)$ (4-round RC6 with Type I weak key)

\begin{tabular}{l|cccccccccccccccccccccccccccc}
\hline Input mask & 00 & 00 & 00 & 00 & 00 & 00 & 00 & 00 & 00 & 00 & 00 & 00 & 00 & 00 & 00 & 00 & 00 & 00 & 00 & 00 & 00 & 00 & 00 & 00 & 00 \\
\hline 1st R. Bias $\left(\times 2^{-10}\right)$ & 1 & -1 & 1 & -1 & 1 & -1 & 1 & -1 & 1 & -1 & 1 & -1 & 1 & -1 & 1 & -1 & 1 & -1 & 1 & -1 & 1 & -1 & 1 & -1 & 1 \\
2nd R. mask & 0 & 01 & 02 & 03 & 04 & 10 & 11 & 12 & 13 & 14 & 20 & 21 & 22 & 23 & 24 & 30 & 31 & 32 & 33 & 34 & 40 & 41 & 42 & 43 & 44 \\
3rd R. Bias $\left(\times 2^{-10}\right)$ & 1 & -1 & 1 & -1 & 1 & -1 & 1 & 1 & -1 & -1 & 1 & 1 & 1 & 1 & 1 & -1 & -1 & 1 & 1 & 1 & 1 & -1 & 1 & 1 & 1 \\
\hline Output mask & 00 & 00 & 00 & 00 & 00 & 00 & 00 & 00 & 00 & 00 & 00 & 00 & 00 & 00 & 00 & 00 & 00 & 00 & 00 & 00 & 00 & 00 & 00 & 00 & 00 \\
\hline \hline Total Bias $\left(\times 2^{-20}\right)$ & 1 & 1 & 1 & 1 & 1 & 1 & 1 & -1 & -1 & 1 & 1 & -1 & 1 & -1 & 1 & 1 & -1 & -1 & 1 & -1 & 1 & 1 & 1 & -1 & 1 \\
\hline
\end{tabular}

Table 7. Matrix $M$ of the bias $\left(\times 2^{-10}\right)$ of linear equations for 2-round RC6 with weak key

\begin{tabular}{|c|c|c|c|c|c|c|c|c|c|c|c|c|c|c|c|c|c|c|c|c|c|c|c|c|c|}
\hline \multirow{2}{*}{$\begin{array}{c}\text { Input } \\
(i, j)\end{array}$} & \multicolumn{25}{|c|}{ Output Mask $(k, l)$} \\
\hline & 00 & 01 & 02 & 03 & 04 & 10 & 11 & 12 & 13 & 14 & 20 & 21 & 22 & 23 & 24 & 30 & 31 & 32 & 33 & 34 & 40 & 41 & 42 & 43 & 44 \\
\hline 00 & 1 & -1 & 1 & -1 & 1 & -1 & 1 & -1 & 1 & -1 & 1 & -1 & 1 & -1 & 1 & -1 & 1 & -1 & 1 & -1 & 1 & -1 & 1 & -1 & 1 \\
\hline 01 & -1 & 1 & -1 & 1 & -1 & -1 & 1 & -1 & 1 & -1 & 1 & -1 & 1 & -1 & 1 & 1 & -1 & 1 & -1 & 1 & -1 & 1 & -1 & 1 & -1 \\
\hline 02 & 1 & -1 & 1 & -1 & 1 & 1 & -1 & 1 & -1 & 1 & 1 & -1 & 1 & -1 & 1 & 1 & -1 & 1 & -1 & 1 & -1 & 1 & -1 & 1 & -1 \\
\hline 03 & -1 & 1 & -1 & 1 & -1 & -1 & 1 & -1 & 1 & -1 & -1 & 1 & -1 & 1 & -1 & -1 & 1 & -1 & 1 & -1 & -1 & 1 & -1 & 1 & -1 \\
\hline 04 & 1 & -1 & 1 & -1 & 1 & 1 & -1 & 1 & -1 & 1 & 1 & -1 & 1 & -1 & 1 & 1 & -1 & 1 & -1 & 1 & 1 & -1 & 1 & -1 & 1 \\
\hline 10 & -1 & -1 & 1 & 1 & -1 & 1 & 1 & -1 & -1 & 1 & -1 & -1 & 1 & 1 & -1 & 1 & 1 & -1 & -1 & 1 & -1 & -1 & 1 & 1 & -1 \\
\hline 11 & 1 & -1 & -1 & 1 & 1 & -1 & 1 & 1 & -1 & -1 & -1 & 1 & 1 & -1 & -1 & 1 & -1 & -1 & 1 & 1 & 1 & -1 & -1 & 1 & 1 \\
\hline 12 & 1 & 1 & -1 & -1 & 1 & -1 & -1 & 1 & 1 & -1 & -1 & -1 & 1 & 1 & -1 & -1 & -1 & 1 & 1 & -1 & -1 & -1 & 1 & 1 & -1 \\
\hline 13 & -1 & 1 & 1 & -1 & -1 & 1 & -1 & -1 & 1 & 1 & 1 & -1 & -1 & 1 & 1 & 1 & -1 & -1 & 1 & 1 & 1 & -1 & -1 & 1 & 1 \\
\hline 14 & -1 & -1 & 1 & 1 & -1 & 1 & 1 & -1 & -1 & 1 & 1 & 1 & -1 & -1 & 1 & 1 & 1 & -1 & -1 & 1 & 1 & 1 & -1 & -1 & 1 \\
\hline 20 & 1 & 1 & 1 & 1 & -1 & -1 & -1 & -1 & -1 & 1 & 1 & 1 & 1 & 1 & -1 & -1 & -1 & -1 & -1 & 1 & 1 & 1 & 1 & 1 & -1 \\
\hline 21 & 1 & -1 & -1 & -1 & -1 & 1 & -1 & -1 & -1 & -1 & -1 & 1 & 1 & 1 & 1 & -1 & 1 & 1 & 1 & 1 & 1 & -1 & -1 & -1 & -1 \\
\hline 22 & 1 & 1 & -1 & -1 & -1 & 1 & 1 & -1 & -1 & -1 & -1 & -1 & 1 & 1 & 1 & -1 & -1 & 1 & 1 & 1 & -1 & -1 & 1 & 1 & 1 \\
\hline 23 & 1 & 1 & 1 & -1 & -1 & 1 & 1 & 1 & -1 & -1 & -1 & -1 & -1 & 1 & 1 & -1 & -1 & -1 & 1 & 1 & -1 & -1 & -1 & 1 & 1 \\
\hline 24 & 1 & 1 & 1 & 1 & -1 & 1 & 1 & 1 & 1 & -1 & -1 & -1 & -1 & -1 & 1 & -1 & -1 & -1 & -1 & 1 & -1 & -1 & -1 & -1 & 1 \\
\hline 30 & -1 & -1 & -1 & -1 & -1 & 1 & 1 & 1 & 1 & 1 & -1 & -1 & -1 & -1 & -1 & 1 & 1 & 1 & 1 & 1 & -1 & -1 & -1 & -1 & -1 \\
\hline 31 & -1 & 1 & 1 & 1 & 1 & 1 & -1 & -1 & -1 & -1 & 1 & -1 & -1 & -1 & -1 & -1 & 1 & 1 & 1 & 1 & -1 & 1 & 1 & 1 & 1 \\
\hline 32 & 1 & 1 & -1 & -1 & -1 & 1 & 1 & -1 & -1 & -1 & 1 & 1 & -1 & -1 & -1 & -1 & -1 & 1 & 1 & 1 & -1 & -1 & 1 & 1 & 1 \\
\hline 33 & 1 & 1 & 1 & -1 & -1 & 1 & 1 & 1 & -1 & -1 & 1 & 1 & 1 & -1 & -1 & -1 & -1 & -1 & 1 & 1 & -1 & -1 & -1 & 1 & 1 \\
\hline 34 & 1 & 1 & 1 & 1 & -1 & 1 & 1 & 1 & 1 & -1 & 1 & 1 & 1 & 1 & -1 & -1 & -1 & -1 & -1 & 1 & -1 & -1 & -1 & -1 & 1 \\
\hline 40 & 1 & 1 & 1 & 1 & 1 & -1 & -1 & -1 & -1 & -1 & 1 & 1 & 1 & 1 & 1 & -1 & -1 & -1 & -1 & -1 & 1 & 1 & 1 & 1 & 1 \\
\hline 41 & -1 & 1 & 1 & 1 & 1 & -1 & 1 & 1 & 1 & 1 & 1 & -1 & -1 & -1 & -1 & 1 & -1 & -1 & -1 & -1 & -1 & 1 & 1 & 1 & 1 \\
\hline 42 & 1 & 1 & -1 & -1 & -1 & 1 & 1 & -1 & -1 & -1 & 1 & 1 & -1 & -1 & -1 & 1 & 1 & -1 & -1 & -1 & -1 & -1 & 1 & 1 & 1 \\
\hline 43 & 1 & 1 & 1 & -1 & -1 & 1 & 1 & 1 & -1 & -1 & 1 & 1 & 1 & -1 & -1 & 1 & 1 & 1 & -1 & -1 & -1 & -1 & -1 & 1 & 1 \\
\hline 44 & 1 & 1 & 1 & 1 & -1 & 1 & 1 & 1 & 1 & -1 & 1 & 1 & 1 & 1 & -1 & 1 & 1 & 1 & 1 & -1 & -1 & -1 & -1 & -1 & 1 \\
\hline
\end{tabular}

The number of linear characteristic for paths through $\left(e_{s}, e_{t}, 0,0\right)$ in the second round is totally $5^{2}=25$. Among them, there are 17 positive $\left(=2^{-10}\right)$ bias of linear characteristics and 8 negative $\left(=-2^{-10}\right)$ bias of linear characteristics. By taking account of the sign of linear characteristic of each path, we obtain the linear characteristic and the linear probability as follows. (The validity of this observation is demonstrated by computer experiments.)

$$
\begin{gathered}
\operatorname{Bias}_{R C 6(\text { Type I weak key })}\left(\left(e_{0}, e_{0}, 0,0\right) \rightarrow\left(e_{0}, e_{0}, 0,0\right)\right) \\
=(17-8) \cdot\left(2^{-10} 2^{-10}\right)=2^{-16.83} \\
L P_{R C 6 \text { (Type I weak key })}\left(\left(e_{0}, e_{0}, 0,0\right) \rightarrow\left(e_{0}, e_{0}, 0,0\right)\right)=2^{-33.66}
\end{gathered}
$$

We note that linear probability obtained here is much higher than the linear probability of RC6 with average keys $\left(2^{-38}\right)$ and of $\operatorname{RC} 6 \oplus\left(2^{-35.356}\right)$.

Next, we generalize the above method to calculate precisely the linear probability of the input mask and the output mask pattern $\left(e_{i}, e_{j}, 0,0\right) \rightarrow\left(e_{k}, e_{l}, 0,0\right)$ for $2 r$-round RC6 with Type I weak key.

For $i, j, k, l \in\{0, \ldots, 4\}$, let $m=(k-i)(\bmod 32), n=(l-j)(\bmod 32)$. We consider the $25 \times 25$ matrix $M=\left(a_{(i j)(k l)}\right)$ such that $a_{(i j)(k l)}=(-1)^{n \cdot e_{i} \oplus m \cdot e_{j}}$. (See Table [1) Then, the bias of linear equation of $2 r$-round RC6 with Type I weak key can be calculated as follows. 
Table 8 . The bias $\left(\times 2^{-4}\right)$ of linear equation for addition $(+)$

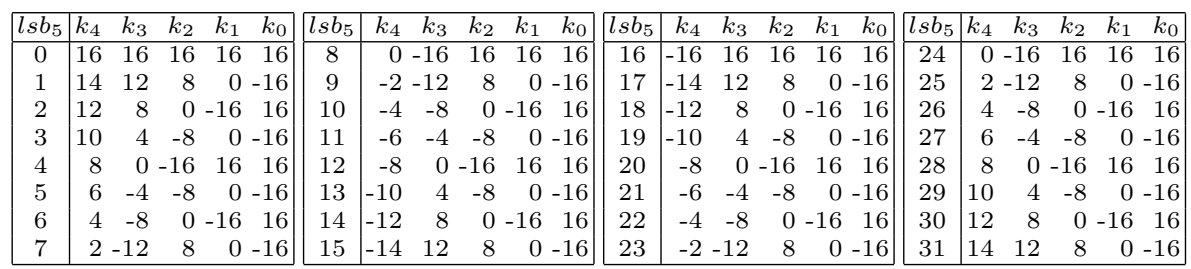

Table 9. The maximum linear probability of RC6 with Type I weak key

\begin{tabular}{c|c|r|r|r|r}
\hline Rounds & $\begin{array}{c}\text { Input Mask } \\
(i, j)\end{array}$ & $\begin{array}{r}\text { Output Mask } \\
(k, l)\end{array}$ & Bias & $L P$ & comment \\
\hline 3 & $(*, *)$ & $(*, *)$ & $1 \cdot 2^{-10}$ & $2^{-20}$ & 1 in $2^{20}$ \\
5 & $(3,8)$ & $(2,2)$ & $21 \cdot 2^{-20}$ & $2^{-31.214}$ & 1 in $2^{30}$ \\
7 & $(1,1)$ & $(3,3)$ & $101 \cdot 2^{-30}$ & $2^{-46.682}$ & 1 in $2^{40}$ \\
9 & $(1,1)$ & $(0,0)$ & $633 \cdot 2^{-40}$ & $2^{-61.386}$ & 1 in $2^{50}$ \\
11 & $(1,1)$ & $(2,2)$ & $4449 \cdot 2^{-50}$ & $2^{-75.760}$ & 1 in $2^{60}$ \\
13 & $(2,2)$ & $(2,2)$ & $24798 \cdot 2^{-60}$ & $2^{-90.804}$ & 1 in $2^{70}$ \\
15 & $(2,2)$ & $(0,0)$ & $134645 \cdot 2^{-70}$ & $2^{-105.922}$ & 1 in $2^{80}$ \\
17 & $(2,2)$ & $(0,0)$ & $942657 \cdot 2^{-80}$ & $2^{-120.306}$ & 1 in $2^{90}$ \\
\hline
\end{tabular}

$$
\begin{gathered}
\Phi^{(r)}=2^{-10 r} M^{r}, \\
\operatorname{Bias}_{R C 6(\text { Type I weak key })_{(2 r)}}\left(\left(e_{i}, e_{j}, 0,0\right) \rightarrow\left(e_{k}, e_{l}, 0,0\right)\right)=\Phi_{(i j)(k l)}^{(r)},
\end{gathered}
$$

where $M^{r}$ means the exponentiation of the integer matrix $M$.

In case of RC6 with arbitrary key, for the least significant five bits of extended key $l s b_{5}(S[4 i-2]), l s b_{5}(S[4 i-1])$, we calculate $\left(k_{i 4}, k_{i 3}, k_{i 2}, k_{i 1}, k_{i 0}\right)$ and $\left(h_{i 4}\right.$, $\left.h_{i 3}, h_{i 2}, h_{i 1}, h_{i 0}\right)$ by using Table 8 and also calculate the following matrix.

$$
K_{i}=\operatorname{diag}\left(k_{i 0} h_{i 0}, k_{i 0} h_{i 1}, \ldots ., k_{i 4} h_{i 4}\right),
$$

where $\operatorname{diag}\left(a_{0}, a_{1}, \ldots\right)$ is the $25 \times 25$ matrix whose diagonal elements are $a_{0}, a_{1}, \ldots$ and other elements are all 0 . For example, when $l s b_{5}(S[2])=4$ and $l s b_{5}(S[3])=$ 26 , then $K_{1}$ is calculated as follows.

$$
K_{1}=\operatorname{diag}\left(2^{-3},-2^{-2}, 0,-2^{-1}, 2^{-1}, 0,0,0,0,0,-2^{-2}, 2^{-1}, \ldots,-1,1\right)
$$

Then, the bias of linear equation of $2 r$-round RC6 can be calculated by using a "Generalized Piling up lemma" of matrix representation as follows.

$$
\begin{gathered}
\operatorname{Bias}_{R C 6_{(2 r)}}\left(\left(e_{i}, e_{j}, 0,0\right) \rightarrow\left(e_{k}, e_{l}, 0,0\right)\right) \\
=\Psi^{(r)}[K]_{(i j)(k l)} \\
\Psi^{(r)}[K]=\prod_{i=1}^{r}\left(2^{-10} M \cdot K_{i}\right)
\end{gathered}
$$

Table 9 shows linear masks that take the maximum linear probability of RC6 with Type I weak key in the elements of $25 \times 25$ matrix calculated as above. Now, we can get the maximal linear probability of $(2 r+1)$-round RC6 with the weak keys by combining the discussion in the case of $2 r$-round RC6 and one-round addition to the input side. 
Table 10. Linear approximations of $2 r$-round RC6 for multiple linear approximation

\begin{tabular}{ccc}
\hline linear approximation & linear probability & number \\
\hline$B e_{0} \oplus D e_{0} \oplus A^{\prime} e_{0} \oplus C^{\prime} e_{0}=0$ & $2^{-20-18(r-1)}$ & 1 \\
$B e_{i} \oplus D e_{0} \oplus A^{\prime} e_{0} \oplus C^{\prime} e_{0}=0$ & $2^{-22-18(r-1)}$ & 4 \\
$B e_{0} \oplus D e_{j} \oplus A^{\prime} e_{0} \oplus C^{\prime} e_{0}=0$ & $2^{-22-18(r-1)}$ & 4 \\
$B e_{0} \oplus D e_{0} \oplus A^{\prime} e_{k} \oplus C^{\prime} e_{0}=0$ & $2^{-22-18(r-1)}$ & 4 \\
$B e_{0} \oplus D e_{0} \oplus A^{\prime} e_{0} \oplus C^{\prime} e_{l}=0$ & $2^{-22-18(r-1)}$ & 4 \\
\hline
\end{tabular}

\section{Multiple Linear Approximation of RC6}

"Multiple Linear Approximation", which is proposed by Kaliski and Robshaw, is a technique to enable to attack ciphers using less amount of data. This technique is quite effective if there exist several linear approximations that have almost maximum linear probability.

Let $\epsilon_{i}$ be the bias of linear equation $L_{i}: X \Gamma_{X_{i}} \oplus Y \Gamma_{Y_{i}}=0,(i=1, \ldots, n)$ with respect to $Y=F(X)$. Then we define weight according to $\epsilon_{i}$ as being $w_{i}=\epsilon_{i} /\left(\epsilon_{0}+\ldots+\epsilon_{n}\right)$. Let $N$ be the number of known plaintexts and $N_{i}$ the number of known plaintexts that satisfy linear approximation $L_{i}$. Then by utilizing the difference between $w_{i} N_{i}$ and $N / 2$ it is not hard to distinguish a cipher from random permutations. The necessary number $N$ of known plaintexts to distinguish a cipher from random permutations is $C /\left(\sum_{i=1}^{n} \epsilon_{i}^{2}\right)$, where $C$ is a parameter which determines the success probability (e.g., $C=4$ implies that the success probability is $95 \%$ ).

By careful consideration of multiple linear approximation, we can see that it is sufficient for estimating necessary number of plaintexts to break a cipher that we get linear approximations whose linear equations are linearly independent. Recall the linear approximations which are discussed in the previous section. The linear approximations we should consider are all of the form $B e_{i} \oplus D e_{j} \oplus A^{\prime} e_{k} \oplus C^{\prime} e_{l}=0$. It is not difficult to see that there are at most 17 linearly independent linear approximations. We utilize 17 linear approximations (shown in Table 10), which are linearly independent and whose linear probabilities are comparatively high, in order to improve the efficiency of breaking RC6.

We estimate the necessary number $N$ of plaintexts to distinguish RC6 from random permutations by applying linear approximations shown in Table 10 to the technique of multiple linear approximation. We note that the coefficients in the equations below are introduced in order to increase the success probability up to $95 \%$.

$$
\begin{aligned}
N & =4 \cdot\left(1 /\left(2^{-20-18(r-1)}+16 \cdot 2^{-22-18(r-1)}\right)\right) \\
& =4 \cdot 1 /\left(\left(1+2^{-2}\right) \cdot 2^{-18 r}\right)=2^{3.68+18 r}
\end{aligned}
$$

Similarly, we estimate the necessary number $N$ of plaintexts to distinguish RC6 $\oplus$ (or RC6 with Type II weak key) from random permutations by seeing Table 3.

$$
N=4 /\left(17 \cdot 2^{-20-15.356(r-1)}\right)=2^{2.556+(15.356) r}
$$


Table 11. Distinguishing attack of RC6

\begin{tabular}{c|c|ll|l}
\hline round & RC6 & RC6(Type II weak key) & RC6(Type I weak key) \\
\hline 3 & $2^{21.68}$ & $2^{17.912}\left(1\right.$ in $\left.2^{16}\right)$ & $2^{17.912}\left(1\right.$ in $\left.2^{20}\right)$ \\
5 & $2^{39.68}$ & $2^{33.268}$ & $\left(1\right.$ in $\left.2^{24}\right)$ & $2^{30.1261}\left(1\right.$ in $\left.2^{30}\right)$ \\
7 & $2^{57.68}$ & $2^{48.624}\left(1\right.$ in $\left.2^{32}\right)$ & $2^{45.712}\left(1\right.$ in $\left.2^{40}\right)$ \\
9 & $2^{75.68}$ & $2^{63.980}\left(1\right.$ in $\left.2^{40}\right)$ & $2^{60.260}\left(1\right.$ in $\left.2^{50}\right)$ \\
11 & $2^{93.68}$ & $2^{79.336}\left(1\right.$ in $\left.2^{48}\right)$ & $2^{75.111}\left(1\right.$ in $\left.2^{60}\right)$ \\
13 & $2^{111.68}$ & $2^{94.692}\left(1\right.$ in $\left.2^{56}\right)$ & $2^{90.061}\left(1\right.$ in $\left.2^{70}\right)$ \\
15 & $2^{129.68}$ & $2^{110.048}\left(1\right.$ in $\left.2^{63}\right)$ & $2^{104.701}\left(1\right.$ in $\left.2^{80}\right)$ \\
17 & $2^{147.68}$ & $2^{125.404}\left(1\right.$ in $\left.2^{70}\right)$ & $2^{119.423}\left(1\right.$ in $\left.2^{90}\right)$ \\
19 & $2^{165.68}$ & $2^{140.760}\left(1\right.$ in $\left.2^{78}\right)$ & $2^{134.227}\left(1\right.$ in $\left.2^{100}\right)$ \\
\hline
\end{tabular}

Moreover, in case of RC6 with Type I weak key, we can pick up linearly independent 17 linear approximations according to the estimation for the linear probability of each input-output masks in Section 8.

Our attacking method, described in this section, is a known plaintext attack. It means that we do not restrict the form of inputs and thus that we can make full use of inputs, that is, the number $2^{128}$ of plaintexts. Therefore, we can say that 13-round RC6 is distinguishable from random permutations and also that 15 -round RC6 $\oplus$, 17-round RC6 with weak keys whose fraction is $2^{-90}$ is distinguishable from random permutations. We summarize these results in Table [1]

\section{Key Recovery of RC6}

In this section, we consider the key recovery of $(2 r+2)$-round RC6 by utilizing the distinguishability result of $(2 r+1)$-round RC6. We adopt a typical method "1-round elimination attack" as same as the method by Knudsen and Meier [13] for key recovery in the following: apply multiple linear approximation to RC6 through the 2 nd round to the $2 r+2$ th round, search exhaustively for the extended key $S[0], S[1]$ (64 bits in total), and use them and the value in the position before key addition of the first round to find a target key. (See Figure 3.)

Since the necessary data size for distinguishing attacker to success the attack is $4 p^{-1}$ with probability $95 \%$, (which is calculated using the linear approximation with the linear probability $p$ ), we claim by our experience that we need the number $4 n p^{-1}$ of known plaintexts and the number $4 p^{-1} 2^{n}$ of computation of the round-function for the number $n(=64)$ of the target key bits with probability $95 \%$ by the one-round elimination method. Thus, we can summarize the necessary data size and complexity to find the target extended key by the one-round elimination method in Table 12

Thus we conclude that the 64-bit target extended key of 14-round RC6 can be recovered with probability $95 \%$ by Multiple Linear Attack with the number $2^{119.68}$ of known plaintexts and the number $2^{185.68}$ of computation of the roundfunction. Also that the 64-bit target extended key of 18-round RC6 with weak key, (the fraction is $2^{-90}$ ), can be recovered with probability $95 \%$ by Multiple 


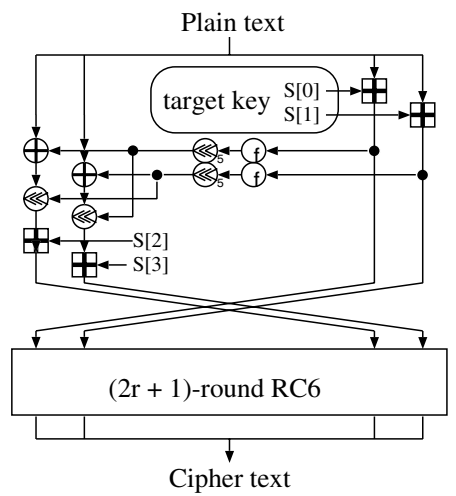

Fig. 3. 1-round elimination attack

Table 12. Key recovery of RC6

\begin{tabular}{c|c|c|c|c}
\hline Rounds & Target & \# Texts & Complexity & Comments \\
\hline 4 & RC6 & $2^{29.68}$ & $2^{95.68}$ & \\
6 & RC6 & $2^{47.68}$ & $2^{113.68}$ & \\
8 & RC6 & $2^{65.68}$ & $2^{131.68}$ & \\
10 & RC6 & $2^{83.68}$ & $2^{149.68}$ & \\
12 & RC6 & $2^{101.68}$ & $2^{167.68}$ & \\
14 & RC6 & $2^{119.68}$ & $2^{185.68}$ & \\
16 & RC6 weak key & $2^{118.048}$ & $2^{184.048}$ & 1 in $2^{64}$ (Type II) \\
18 & RC6 weak key & $2^{127.423}$ & $2^{193.423}$ & 1 in $2^{90}$ (Type I) \\
\hline
\end{tabular}

Linear Attack with the number $2^{127.423}$ of known plaintexts and the number of $2^{64}$ of memory, and the number $2^{193.423}$ of computation of the round-function.

\section{References}

1. A. Biryukov and E. Kushilevitz. Improved cryptanalysis of RC5. EUROCRYPT'98, LNCS 1403, pp.85-99, 1998.

2. J. Borst, B. Preneel, and J. Vandewalle. Linear cryptanalysis of RC5 and RC6. FSE'99, LNCS 1636, pp.16-30, 1999.

3. S. Contini, R.L. Rivest, M.J.B. Robshaw, and Y.L. Yin. The security of the RC6 block cipher. v.1.0, August 20, 1998.

Available at http://www.rsasecurity.com/rsalabs/rc6/.

4. S. Contini, R.L. Rivest, M.J.B. Robshaw, and Y.L. Yin. Improved analysis of some simplified variants of RC6. FSE'99, LNCS 1636, pp.1-15, 1999.

5. H. Gilbert, H. Handschuh, A. Joux and S. Vaudenay, A Statistical Attack on RC6. FSE 2000, LNCS 1978, pp.64-74, 2001.

6. M.H. Heys. Linearly weak keys of RC5. IEE Electronic Letters, Vol.33, pp.836-838, 1997.

7. B.S. Kaliski Jr. and M.J.B. Robshaw. Linear cryptanalysis using multiple approximations. CRYPTO'94, LNCS 839, pp.26-39, 1994. 
8. B.S. Kaliski Jr. and M.J.B. Robshaw. Linear cryptanalysis using multiple approximations and FEAL. FSE'94, LNCS 1008, pp.249-264, 1995.

9. B.S. Kaliski Jr. and Y.L. Yin. On differential and linear cryptanalysis of the RC5 encryption algorithm. CRYPTO'95, LNCS 963, pp.171-184, 1995.

10. B.S. Kaliski Jr. and Y.L. Yin. On the security of the RC5 encryption algorithm. Available at http://www.rsasecurity.com/rsalabs/rc6/.

11. J. Kelsey, B. Schneier, and D. Wagner. Mod $n$ cryptanalysis, with applications against RC5P and M6. FSE'99, LNCS 1363, pp.139-155, 1999.

12. L.R. Knudsen and M.J.B. Robshaw. Non-linear approximations in linear cryptanalysis. EUROCRYPT'96, LNCS 1070, pp.224-236, 1996.

13. L.R. Knudsen and W. Meier. Correlations in RC6 with a reduced number of rounds. FSE 2000, LNCS 1978, pp.94-108, 2001.

14. M. Matsui. Linear cryptanalysis method for DES cipher. EUROCRYPT'93, LNCS 765, pp.386-397, 1993.

15. S. Moriai, K. Aoki and K. Ohta. Key-dependency of linear probability of RC5. IEICE Transactions on Fundamentals of Electronics, Communications and Computer Sciences, Vol.E80-A, No.1, 1997.

16. R.L. Rivest. The RC5 encryption algorithm. FSE'94, LNCS 1008, pp.86-96, 1995.

17. R.L. Rivest, M.J.B. Robshaw, R. Sidney and Y.L. Yin. The RC6 block cipher. v1.1, August 20, 1998. Available at http://www.rsasecurity.com/rsalabs/rc6/.

18. K. Nyberg. Linear approximation of block ciphers. EUROCRYPT'94, LNCS 950, pp.439-444, 1994.

19. A.A. Selcuk. New results in linear cryptanalysis of RC5. FSE'98, LNCS 1372, pp.1-16, 1998. 